\title{
Materials aspects of integrated high Tc dc-SQUID magnetometer fabrication
}

\author{
J.W.M. Hilgenkamp, R.P.J. IJsselsteijn, A.J.H.M. Rijnders, P.A.C. Tavares, J. Flokstra and H. Rogalla.
}

University of Twente, Department of Applied Physics, Low Temperature Division, P.O. Box 217, 7500 AE Enschede, Netherlands.

\begin{abstract}
An integrated high Tc dc-SQUID magnetometer is being developed. It has in total 10 layers of five different materials. Various materials aspects of the fabrication process will be discussed, especially the smoothness of the films and the techniques to obtain smooth edges. Cross-overs and superconducting window contacts were fabricated. The critical temperature of the window-contact is $84 \mathrm{~K}\left(\mathrm{j}_{\mathrm{c}}(77 \mathrm{~K})=2 \cdot 10^{5} \mathrm{~A} / \mathrm{cm}^{2}\right)$ and the resistivity of the insulating $\mathrm{SrTiO}_{3}$ layer in the cross-over is $6 \cdot 10^{5} \Omega \mathrm{cm}$ at $77 \mathrm{~K}$. The complete coil often shows a small resistive component down to about $50 \mathrm{~K}$. Ramp type and bi-epitaxial grain boundary dc-SQUIDs show voltage modulation up to $65 \mathrm{~K}$ and $79 \mathrm{~K}$, respectively. Efforts to fabricate an integrated high $T_{c}$ dc-SQUID magnetometer will be discussed.
\end{abstract}

\section{Introduction}

To increase the field sensitivity of a dc-SQUID use is made of a flux transformer configuration. The flux transformer can be placed on a separate chip [1] or integrated on the same chip as the SQUID $[2,3]$. Since the integrated device consists of a large number of layers good control of layer growth is a prerequisite. Occurrence of outgrowths and holes in the films has to be minimized. Care has to be taken to avoid interface reactions. To provide high quality epitaxial growth of the layers over structures etched in underlying layers, these structures should have smooth edges [4]. Irregular or steep edges may leed to unwanted grain boundaries and even short cuts. Ar ion beam etching under an angle provides these smooth edges. Separate cross-overs, window contacts and multiturn coils have been fabricated to test this etching procedure

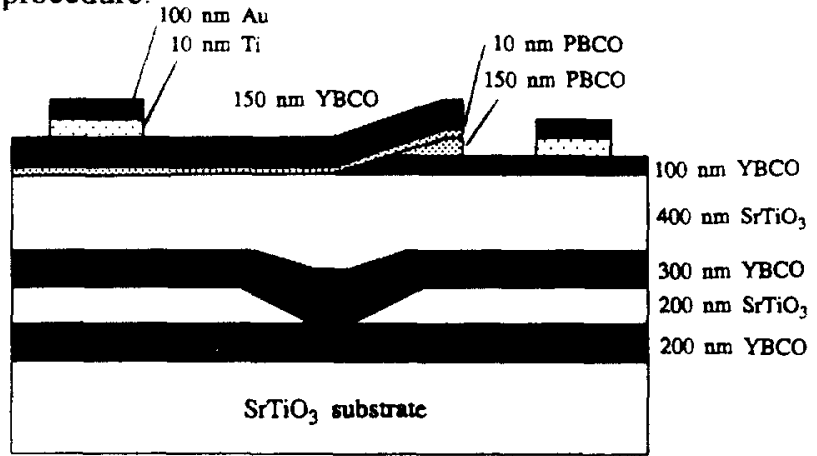

rigure 1: Schematic cross section of the integrated magnetometer.
The key elements of the dc-SQUIDs are the Josephson junctions. Besides good performance (high IcRn-products at high temperatures, low noise levels, stability and reproducibility) they have to be compatible with the techniques used for fabrication of the total device. In our group $\mathrm{YBa}_{2} \mathrm{Cu}_{3} \mathrm{O}_{7-\mathrm{x}}(\mathrm{YBCO}) / \mathrm{PrBa}_{2} \mathrm{Cu}_{3} \mathrm{O}_{7-\mathrm{x}}(\mathrm{PBCO}) / \mathrm{YBCO}$ ramp type junctions and bi-epitaxial grain boundary junctions with various weak link angles are presently investigated.

\section{Design}

In figure 1 the design of the integrated dcSQUID magnetometer is schematically shown.

The coil structure is placed directly on the substrate beneath the SQUID-washer. In this way the fabrication of the junctions is the last step in the process. The coil windings and the returnstrip are separated by a $\mathrm{SrTiO}_{3}$ layer. A window provides a superconducting contact between these layers in the centre of the coil. After structuring the returnstrip of the coil a $400 \mathrm{~nm}$ thick $\mathrm{SrTiO}_{3}$ insulation layer is deposited to separate the coil from the SQUID washer.

The YBCO/PBCO bi-layer that serves as the base electrode of our junctions is deposited in situ. After definition of the junction ramp by photolithography and Ar-ion beam etching under an angle, the ramp is cleaned by Ar ion milling before in situ deposition of the barrier and top layers takes place. In the case when bi-epitaxial grain boundary 
junctions are used an $\mathrm{MgO} / \mathrm{CeO}_{2}$ bi-layer is deposited in situ after the $\mathrm{SrTiO}_{3}$ insulation layer has been deposited. After structuring the $\mathrm{CeO}_{2}$ template layer and cleaning of the edge by $\mathrm{Ar}$ ion milling the YBCO junction layer is deposited in situ.

A contact layer of $\mathrm{Ti}$ and $\mathrm{Au}$ is then deposited by RF magnetron sputtering and structured by lift off before, as a final step, the square washer dcSQUID structures are defined by Ar ion-beam or Ar plasma etching.

The 5 turn input coil has $8 \mu \mathrm{m}$ wide windings, separated $5 \mu \mathrm{m}$ from each other. It is connected to a $3.1 \times 4 \mathrm{~mm}^{2}$ pick up coil or to contact pads. In the latter case the $\mathrm{SrTiO}_{3}$ is removed from the contacts by selective etching with a $2 \%$ HF solution in $\mathrm{H}_{2} \mathrm{O}$ [5] followed by a short Ar plasma cleaning treatment.

The washer has an outer dimension of $150 \mu \mathrm{m} \times$ $155 \mu \mathrm{m}$ and a hole size of $16 \mu \mathrm{m} \times 16 \mu \mathrm{m}$. The inductance $\mathrm{L}$ is estimated to be about $30 \mathrm{pH}$. The slit inductance is partly shielded by placing it over the return strip of the input coil. The junctions have a width of $12 \mu \mathrm{m}$. In the case of the ramp type junctions the critical current can be set to $32 \mu \mathrm{A}$ at the working temperature by adjusting the barrier thickness so that the screening factor $\beta_{\mathrm{L}}\left(=2 \mathrm{LI} \mathrm{c}_{\mathrm{c}} / \phi_{\mathrm{o}}\right)$ equals 1 .

\section{Deposition}

RF magnetron sputtering [6] is used for the junction barrier and top layer as well as the contact layers, and pulsed laser deposition [7] for the other layers. We use $\mathrm{SrTiO}_{3}$ substrates. Yttrium stabilized $\mathrm{ZrO}_{2} \quad$ (YSZ) substrates were not applicable, because after structuring the coil windings subsequent deposition of $\mathrm{SrTiO}_{3}$ resulted in $80 \%$ (110) orientation, inducing mainly (103) oriented YBCO in the return strip. This problem does not occur if $\mathrm{SrTiO}_{3}$ substrates are used.

We have studied the influence of the process parameters of laser deposition on the occurrence of droplets and outgrowths in YBCO films [8]. The droplet density is minimal when a laser fluence below about $1.0 \mathrm{~J} / \mathrm{cm}^{2}$ is used. The outgrowth density decreases with increasing laser pulse rate or decreasing deposition temperature. High quality smooth films, with an outgrowth density below 1 per $100 \mathrm{~m}^{2}$, were obtained at a rate of $10 \mathrm{~Hz}$ and a deposition temperature of $720^{\circ} \mathrm{C}$. The oxygen pressure is $25 \mathrm{~Pa}$. Large differences in the smoothness of $\mathrm{YBCO} / \mathrm{SrTiO}_{3}$ bi-layers caused by only small changes in deposition temperature were observed. The difference of the deposition temperatures for a closed smooth surface and for a surface with holes or outgrowths is only $10^{\circ} \mathrm{C}$. We found an optimal deposition temperature of $730^{\circ} \mathrm{C}$ with an oxygen pressure of $15 \mathrm{~Pa}$ and a laser pulse energy of 1.4 $\mathrm{J} / \mathrm{cm}^{2}$. Sputtered YBCO films are deposited at $740^{\circ} \mathrm{C}$ with a sputter pressure of 13Pa. The Ar/oxygen ratio is $3: 2$.

The deposition conditions for YBCO and $\mathrm{PBCO}$ are similar.

The optimal deposition parameters were derived from single, bi- or tri-layer studies. The influence of a larger number of underlying layer on the optimal deposition parameters is studied.

\section{Etching}

To provide epitaxial growth, structures should have edges that are very smooth with an angle of $30^{\circ}$ or less with the substrate surface. Wet chemical methods do not yield these smooth edges and also Ar plasma etching is not suitable.

We use Ar ion beam etching under an angle to obtain low angle edges in our structures. An AZ 1518 photoresist layer is baked out in an oven at $90^{\circ} \mathrm{C}$ for 10 minutes. The energy of the incident ions is $500 \mathrm{eV}$. The beam current is $10 \mathrm{~mA}$ and the angle used is $45^{\circ}$. The structuring is performed intermittently at an $\mathrm{Ar}$ pressure of $5 \cdot 10^{-2} \mathrm{~Pa}$ resulting in an effective etch-rate of $38 \mathrm{~nm} / \mathrm{min}$ for YBCO and $23 \mathrm{~nm} / \mathrm{min}$ for $\mathrm{SrTiO}_{3}$. In one run both edges of a stripline can be etched in the correct way [8].

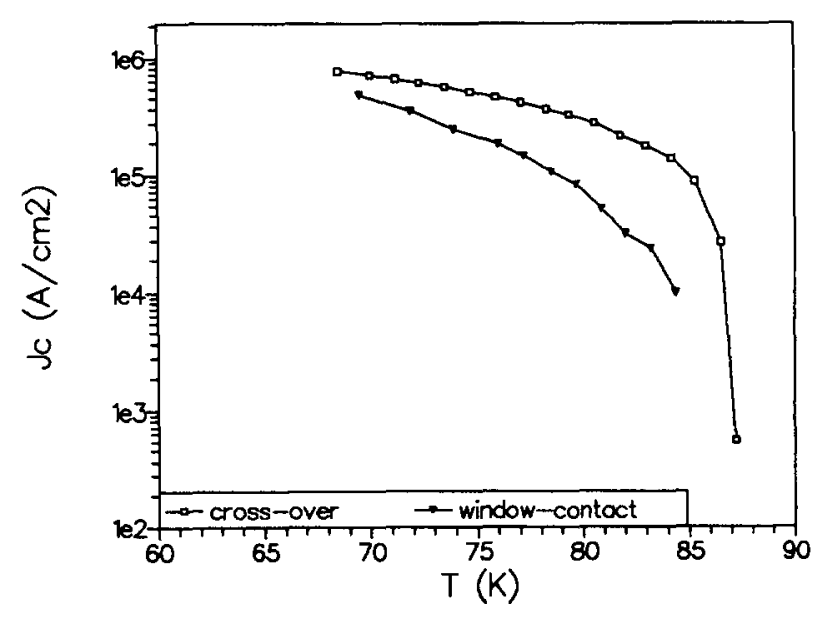

Figure 2: $J_{c}$ as a function of $T$ for the window contact and the top-strip of the cross-over.

Using the Ar ion gun etching procedure separate cross-overs, window contacts and multiturn coils 
have been fabricated. In figure 2 the critical current density of a window contact and the topstrip of a cross-over is shown as a function of $T$.

The base YBCO layer of the cross-over has a thickness of $200 \mathrm{~nm}$ and is etched into a stripline of $150 \mu \mathrm{m}$ width using the ion beam method. On top of this a $150 \mathrm{~nm}(100)$ oriented $\mathrm{SrTiO}_{3}$ layer is grown. The $200 \mathrm{~nm}$ thick YBCO top layer is structured into a $30 \mu \mathrm{m}$ wide stripline using $\mathrm{Ar}$ ion plasma etching. The critical temperature of the top layer is $87 \mathrm{~K}$. $j_{c}$ at $77 \mathrm{~K}$ equals $5 \cdot 10^{5} \mathrm{~A} / \mathrm{cm}^{2}$. The resistivity of the $\mathrm{SrTiO}_{3}$ layer at $77 \mathrm{~K}$ is $6.10^{5}$ $\Omega \mathrm{cm}$. Window contacts were fabricated with a critical temperature of $84 \mathrm{~K}$ and a critical current density of $2 \cdot 10^{5} \mathrm{~A} / \mathrm{cm}^{2}$ at $77 \mathrm{~K}$. The contact has an effective area in the ab-plane of $2.5 \mu \mathrm{m}^{2}$.

The quality of the small number of coils that we have made so far is not as good as that of the separate cross-overs and windows. The best coil had a $\mathrm{T}_{c}$ of $50 \mathrm{~K}$. It is a 12.5 turn coil with a linewidth of $30 \mu \mathrm{m}$. The total length is $4.6 \mathrm{~cm}$. The window-contact is $30 \mu \mathrm{m} \times 60 \mu \mathrm{m}$. We believe that the problem occurs in the return strip of the coil.

\section{Junctions and DC-SQUIDs}

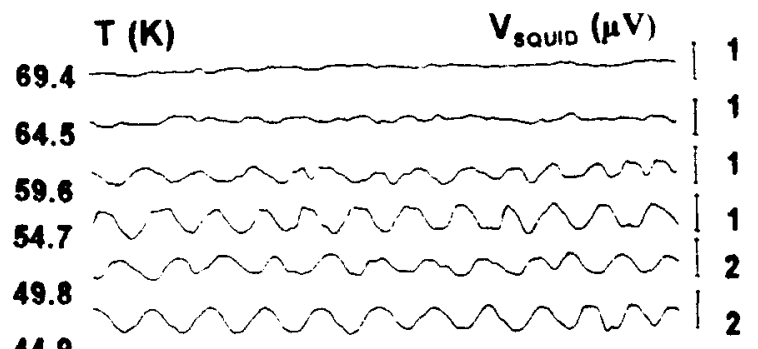

44.9

40.0

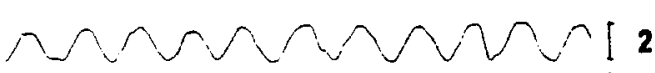

35.1

30.2

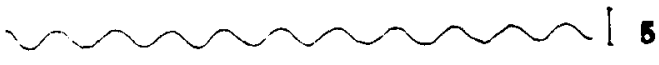

25.3
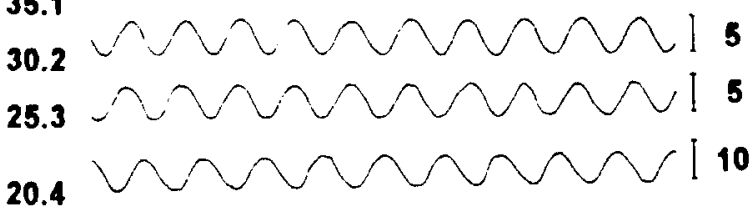

20.4

15.5

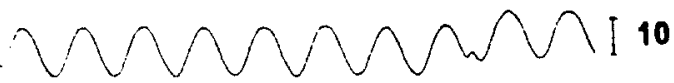

10.8

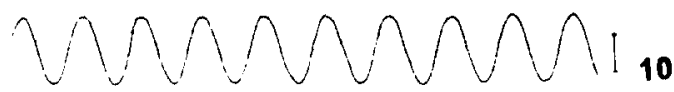

7.4

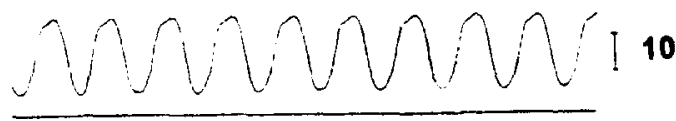

applied field

Figure 3: $V(\phi)$ as a function of temperature for a ramp type DC-SQUID.
$\mathrm{YBCO} / \mathrm{PBCO} / \mathrm{YBCO}$ ramp type junction [9] and biepitaxial grain boundary junctions with weak link angles of $18^{\circ}$ and $27^{\circ}[10]$ are presently studied in our group. The ramp type junctions are characterized by rather high $I_{c} R_{n}$ products, up to $8 \mathrm{mV}$ at $4.2 \mathrm{~K}$. By varying the barrier thickness the critical current of the junctions can more or less be adjusted. $I_{c}$ and $R_{n}$ have been measured as a function of temperature and Shapiro steps have been observed up to $80 \mathrm{~K}[11]$.

DC-SQUIDs based on ramp type junctions showed voltage modulation up to about $65 \mathrm{~K}$. The voltage modulation by an applied magnetic field is shown at various temperatures in figure 3. Nicely periodic behaviour was observed over several thousands of flux quanta.

The noise power spectrum of one of our SQUIDS was measured at $4.2 \mathrm{~K}$. The self-inductance of this sample was about $100 \mathrm{pH}$. The critical current was $20 \mu \mathrm{A}, \beta_{\mathrm{L}}=2$. The white noise level at frequencies above $40 \mathrm{~Hz}$ was $10^{-27} \mathrm{~J} / \mathrm{Hz}$. Below $40 \mathrm{~Hz}$ the noise predominantly has a $1 / \mathrm{f}$ character resulting in a noise level of $8 \cdot 10^{-27} \mathrm{~J} / \mathrm{Hz}$ at $1 \mathrm{~Hz}$. It is not clear however if these high noise levels are structural for these types of junctions or that this is specific for this sample.

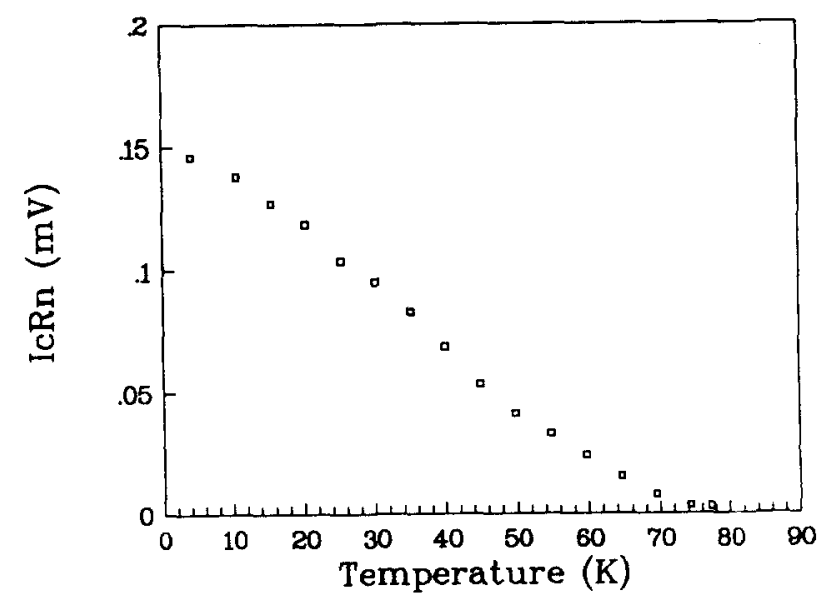

Figure 4: $I_{c} R_{n}$ product as a function of temperature for an $18^{\circ}$ bi-epitaxial grain boundary SQUID.

Bi-epitaxial grain boundary junctions and dcSQUIDs with weak link angles of $18^{\circ}$ and $27^{\circ}$ have been fabricated. The maximum operation temperature for these devices is about $80 \mathrm{~K}$. The temperature dependence of the $I_{c} R_{n}$ product for an $18^{\circ} \mathrm{dc}$-SQUID is given in figure 4 . The junctions have a width of $10 \mu \mathrm{m}$ and a thickness of $200 \mathrm{~nm}$. The $R_{n}$ value is about $1 \Omega$ independent of temperature. The voltage modulation of this SQUID is shown in figure 5 for 
different values of the bias current at $77 \mathrm{~K}$. The maximum modulation depth at this temperature is $1.5 \mu \mathrm{V}$.

The noise level at $4.2 \mathrm{~K}$ was $10^{-28} \mathrm{~J} / \mathrm{Hz}$ at $100 \mathrm{~Hz}$. Below this frequency the noise has a $1 / f$ character.

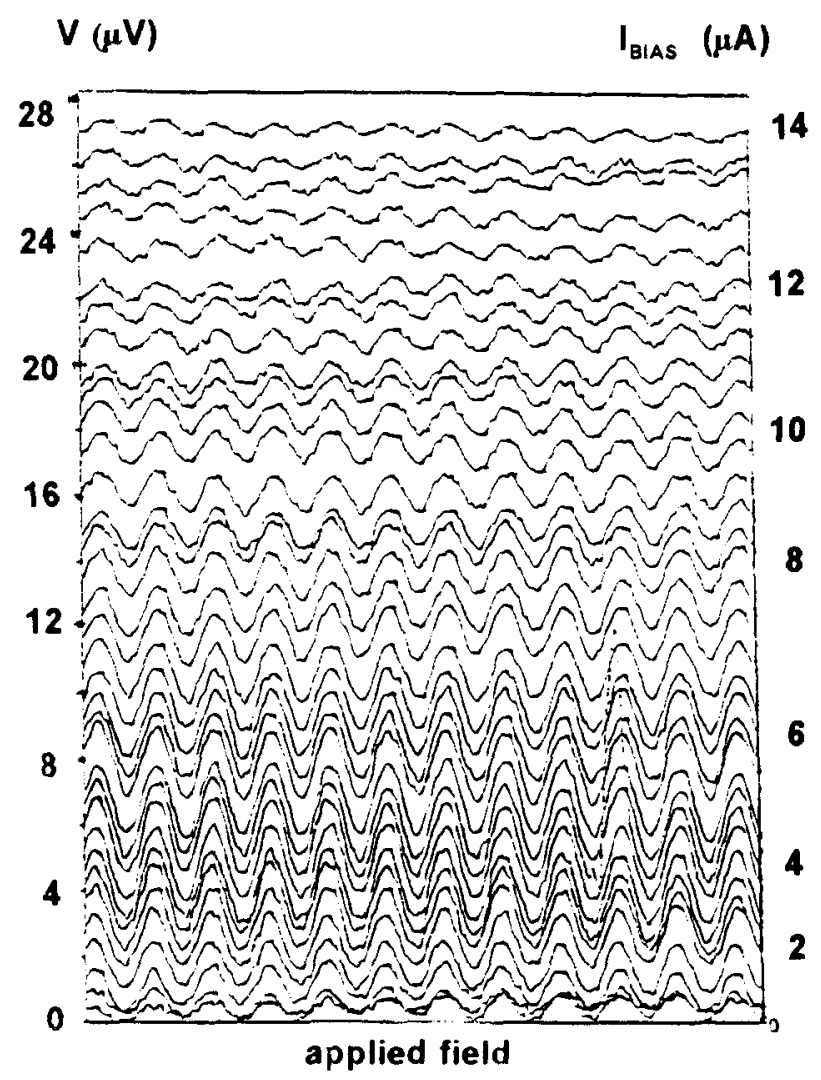

Figure 5: $V(\phi)$ for different values of the bias current for an $18^{\circ}$ bi-epitaxial grain boundary SQUID at $77 \mathrm{~K}$.

The design was however not optimal at this temperature for $\beta_{\mathrm{L}}=10$. Considerable improvement of the noise levels may be expected by the use of a bias reversal method.

\section{Integrated DC-SQUID magnetometer}

Although the basic elements for an integrated dc-SQUID magnetometer could be prepared, a complete integration was not succesful for the first three trials up to now. The coil structure functioned as described but some influence of later processing steps was noticeable. The base electrode on top of the $\mathrm{SrTiO}_{3}$ insulating layer is superconducting at $78 \mathrm{~K}$ but the ramp type junctions did not function adequately. The smoothness of the multilayer structure underneath is probably not good enough. Improvement may be expected when also the junction structures are realised on the substrate. Further work is in progress.

\section{Conclusions}

Several material aspects relevant for the fabrication of an integrated high $\mathrm{T}_{\mathrm{c}}$ dc-SQUID magnetometer have been studied. Using optimal deposition parameters reasonable flat layers could be fabricated. Ar ion beam etching is a fruitfull technique for the fabrication of smooth edges necessary for cross-overs, window contacts and other ramps. High quality window contacts and cross-overs were made but coils often showed a resistive tail down to $50 \mathrm{~K}$. Ramp type and biepitaxial dc-SQUIDs were fabricated with operation temperatures up to $80 \mathrm{~K}$. The complete integration of all elements to an integrated dc-SQUID magnetometer failed up to now.

\section{References}

[1]: F.C. Wellstood, A.H. Miklich, J.J. Kingston, M.J. Ferrari, J. Clarke, M.S. Colclough, K. Char and G. Zaharchuk, SQUID'91 Berlin p. 162

[2]: A.H. Miklich, D. Koelle, E. Dantsker, D.T. Nemeth, J.J. Kingston, R.F. Kromann and J. Clarke, ASC'92, Chicago.

[3]: L.P. Lee, K. Char, M.S. Colclough and G. Zaharchuk, Appl. Phys. Lett. 59, 3051, (1991).

[4]: C.L. Jia, B. Kabius, K. Urban, K. Herrman, G.J. Cui, J.S. Schubert, W. Zandler, A.I. Braginski and C. Heiden, Physica C 171, 545, (1991).

[5]: W. Eidelloth, W.J. Gallagher, R.P. Robertazzi, R.H. Koch, B. Oh, R.L. Sandstrom, Appl. Phys. Lett 59, 1257, (1991).

[6]: J. Gao, B. Häuser and H. Rogalla, J. Appl. Phys. 67, 2512, (1990).

[7]: D.H.A. Blank, D.J. Adelerhof, J. Flokstra and H. Rogalla, Physica C 167, 423, (1990).

[8]: J. Flokstra, R.P.J. IJsselsteijn and J.W.M. Hilgenkamp, Thin Solid Films, 218, 304, (1992).

[9]: Yu. Boguslavskij, J. Gao, A.J.H.M. Rijnders,

D. Terpstra, G.J. Gerritsma and H. Rogalla, Physica C, 194, 268, (1992).

[10]: R.P.J.Jsselsteijn, J.W.M. Hilgenkamp, M. Eisenberg, C. Vittoz, J. Flokstra and H. Rogalla. E-MRS, Strasbourg, fall 1992.

[11]: D. Terpstra, Private Communications. 